\title{
Plan de Investigación del IIES-UCA
}

Nuestro punto de partida es el principio de que la finalidad de todo proceso de desarrollo económico debe ser, el mejoramiento del bienestar material de la población en general. En una sociedad agobiada por la pobreza, ésto significa especialmente el avance sostenido hacia la erradicación de ese fenómeno. Este ha de constituir el horizonte de toda estrategia económica, y el criterio principal del desarrollo económico.

A costa de alguna simplificación, se puede afirmar que en El Salvador esa empresa demanda, en el plano económico, fundamentalmente 2 cosas:

- Un crecimiento económico fuerte y sostenido (acompanado de niveles elevados de empleo).

- Una distribución más equitativa del ingreso y de los frutos del crecimiento.

Esto es asi porque el desarrollo económico de un país no puede reducirse al sólo aumento de la producción de bienes y servicios, ni tampoco a una distribución más equitativa de un ingreso insuficiente. Dicho de otro modo, no todo proceso de crecimiento implica desarrollo, pero todo proceso de desarrollo requiere de crecimiento económico.

También es evidente que entre los objetivos de un crecimiento económico fuerte y de una distribución más equitativa de los frutos del mismo, pueden presentarse conflictos. Las estrategias de desarrollo o los "proyectos de nación" se diferencian fundamentalmente por la forma en que resuelven o plantean resolver esos conflictos.

En lo que a continuación se presenta se discuten algunos pilares que una estrategia de crecimiento fuerte, sostenido y equitativo para EI Salvador deberia de tener. El propósito de ello no es, sin embargo, sehalar lineamientos para to que podria ser una estrategia de tal naturaleza. Más bien, lo que se pretende es identificar posibles temas o 
áreas de investigación que se inscriban en el marco de la problemática de un crecimiento fuerte, sostenido y equitativo. Dado el carácter esquemático de este documento (el documento es más un plan de investigación), tampoco se hacen esfuerzos por sustentar exhaustivamente todos los planteamientos desarrollados. El objetivo fundamental del documento es plantear de una manera clara las reflexiones que enmarcan los temas que se proponen para ser abordados dentro de las áreas básicas de investigación del Instituto.

\section{Crecimlento fuerte y sostenldo}

1. En un pais con un ingreso por habitante tan bajo, como El Salvador, el fenómeno de la pobreza no se explica únicamente por una distribución sumamente inequitativa de la riqueza y del ingreso. El nivel de ingreso, que resulta principalmente de la capacidad productiva de la economía, es también un factor importante en la explicaclón de la pobreza. El Salvador, cuyo ingreso por habitante es actualmente inferior al de hace más de un cuarto de siglo, está obligado no sólo a recuperar el inmenso terreno perdido durante la década pasada, sino también a incrementar aceleradamente el tamanto pre-crisls de su producto. Ello requiere que el pals logre y sostenga en las próximas décadas altas tasas de crecimiento económico. Esta tarea exige, en el plano económico, fundamentalmente lo siguiente:

1.1. La transformación/reconversión sistemática del aparato productivo nacional. Esta transformación, que demanda un mayor esfuerzo en términos de acumulación, implica principalmente lo siguiente:

- La incorporación (orientada) y adaptación de los avances tecnológicos que se han registrado en el mundo en los últimos antos.

- El desarrollo de capacidades tecnológicas propias.

El primer aspecto, especialmente, está asociado a la reconversión del aparato productivo industrial, asi como al mejoramiento de las técnicas de producción en el sector agropecuario, factores claves para la elevación de la productividad que un crecimiento fuerte y sostenido exige. El segundo se vincula fundamentalmente al fomento que deberla darse a las actividades de investigación y desarrollo, al fomento de la ciencia y tecnología, con el objeto de elevar la productividad de nuestra economia.

Ambos aspectos implican la definición y adopción de una polltica tecnológica sistemática y deliberada. Puesto que es muy dificil que ésto se realice espontáneamente en la escala y modalidad que la economla salvadoreha demanda - al menos esto es lo que la experiencia en el 
pais ha puesto en evidencia-, en esta tarea el Estado debería de desempenar un rol importante, al menos como promotor y orientador del proceso.

El problema de la tecnología apropiada es un aspecto importante que habrá de abordarse dentro de los esfuerzos de reconversión, particularmente en su relación con la productividad y el empleo.' Esto teniendo en cuenta, especialmente, que pueden existir tecnologias que rindan una elevada productividad, potenciando de esta manera el crecimiento, pero que sin embargo sean excesivamente ahorradoras de mano de obra y contribuyan muy poco a reducir el desempleo o la pobreza.

Por otra parte, debe tenerse en cuenta que dado que es prácticamente imposible lograr una transformación productiva acelerada y total en un plazo relativamente corto, es necesario considerar que la heterogeneidad estructural y el rezago tecnológico de la base productiva seguirán actuando en el corto y mediano plazo como restricciones no sólo para la recuperación económica sino también para un crecimiento equilibrado. La heterogeneidad estructural demandará, en este contexto, de una acción especial.

Debe considerarse, asimismo, que puesto que la economía salvadorena carece de sectores productores de bienes intermedios y de capital lo suficientemente desarrollados, el imperativo de la transformación productiva implica, por otra parte, la necesidad de un intenso esfuerzo de abastecimiento de insumos y bienes de capital, la inmensa mayoria de los cuales habrán de obtenerse fundamentalmente via importaciones, al menos en el corto y mediano plazo. Algo similar es válido también para la tecnología no tangible. Esto plantea al menos dos puntos:

- la necesidad del financiamiento externo. Esto conduce a que tenga que abordarse problemas como el de la inversión extranjera directa, el endeudamiento externo, y la ayuda externa. El entorno internacional y los cambios geopolíticos son importantes restricciones a considerar en ésto.

1. La relación entre tecnologla y ecologla serla también otro punto a tener en cuenta en la definición de la tecnologla apropiada, particularmente si se tiene en mente la sostenibilidad del crecimiento y la subordinación de éste a la elevación del bienestar de la población. En este documento, sin embargo, este elemento no se considera; tampoco se considera el problema ecológico en general. Creemos, sin embargo, que ésta es una dimensión de gran importancia que deberla ser abordada desde un enfoque multidisciplinario, de cara al diseño de un plan integral de desarrollo. 
- la necesidad de fortalecer la capacidad exportadora del pals. Para ello es imprescindible la mejora de nuestra competitividad en los mercados mundiales y la adopción de una polltica deliberada de promoción de exportaciones. La transformación productiva ha de ser a su vez un componente fundamental de esta politica y una $\infty$ ndición para el mejoramiento sostenido de nuestra competitividad. Las políticas cambiaria y comercial, etc. son también aspectos de gran importancia.

El problema del sector externo merece una mención especial. El Salvador ha sido, y será por un buen tiempo, un pals con un alto grado de vulnerabilidad extema. A los límites tradicionales de escasa diversificación de las exportaciones y rigidez de la estructura de importaciones se ha anadido en los últimos anos la disminución del volumen de exportaciones, el peso de la deuda extema y el deterioro de los términos de intercambio. Aunque ésto ha sido superado en alguna medida con la ayuda de las transferencias privadas (remesas) y las donaciones oficiales, ello ha reforzado la dependencia de la capacidad de importación del país de variables totalmente exógenas al desempeno exportador de nuestro aparato productivo. Por otra parte, no puede pensarse en ellas como una fuente permamente de divisas.

Dado que en una economla como la nuestra (pequefa y abierta) el ritmo de crecimiento económico es fuertemente afectado por la disponibilidad de divisas, la fortaleza o fragilidad del sector extemo se convierte en un factor de est/mulo o restricción para crecer sostenida o establemente. En este sentido, cualquier estrategia de crecimiento debe tener muy en cuenta la viabilidad del sector extemo.

1.2. El fortalecimiento de la capacidad de acumulación de capital. Esto supone fundamentalmente un incremento del coeficiente de ahorro e inversión nacional. La modernización y fortalecimiento del sistema financiero nacional -y la promoción de pautas de consumo más austeras donde ésto sea posible-, por una parte, y el financiamiento externo (o el ahorro externo), por otra, son elementos esenciales al respecto. Los problemas de la inversión extranjera y del endeudamiento externo aparecen nuevamente acá. Las pollticas tributaria y de tasas de interés habrán de constituir también elementos importantes de cara a este esfuerzo. El incremento del ahorro público, por su parte, tampoco carece de relevancia en este contexto.

1.3. La realización de mayores y renovados esfuerzos en la formación de capital humano. Este es un elemento importante tanto para el crecimiento en sl como en la tarea de transformación de la estructura productiva, y demanda de la definición y adopción de una estrategia 
sistemática y deliberada de formación, adiestramiento, readiestramiento, etc. de recursos humanos. La naturaleza misma de la tarea y el bajo nivel de educación y adiestramiento técnico de la población salvadorena, indican que el Estado deberá jugar un rol importante en ese esfuerzo. La readecuación/reforma del sistema educativo es un aspecto que habrá de abordarse dentro de estos esfuerzos. Debe recordarse a este respecto que el problema no se reduce simplemente a la deseabilidad de la educación, sino también a qué clase de educación es la más adecuada para promover la transformación productiva, el crecimiento y la equidad.

La necesidad de elevar/mejorar la capacidad productiva ffsica del pals, a fin de dotar de una base firme al proceso fuerte y sostenido de crecimiento, se encuentra detrás de los tres puntos anteriores.

1.4. Mayor eficiencia en el uso de los recursos. Una mayor eficiencia permitirla incrementar el tamaño del producto para un nivel dado de capacidad productiva física (fuerza de trabajo y medios de producción), es decir, incrementar la productividad de los recursos. Esta tarea es algo que no atafie solamente al sector público, aunque en éste una mayor eficiencia constituye un imperativo impostergable. El logro de este objetivo exige en general afrontar una serie de distorsiones acumuladas en nuestra economia, así como la realización de reformas institucionales que involucran en buena medida -aunque de ningún modo de manera exclusiva - al sector público. El avance hacia la transformación productiva ha de preceder al hacer frente a algunas de esas distorsiones, sobre todo las que tienen que ver con la política $c o-$ mercial.

1.5. Un contexto macroeconómico estable. Esto significa fundamentalmente una inflación razonablemente baja (digamos la exigida por los cambios en los precios relativos) y un déficit externo sostenible en el mediano-largo plazo. ${ }^{2}$ Ambos aspectos están íntimamente relacionados con el manejo de las políticas fiscal, monetario-crediticia y cambiaria, asI como con la política financiera internacional. La transformación productiva, en tanto propiciadora de una mayor competitividad, aparece nuevamente acá como elemento importante, particularmente con relación al segundo aspecto.

2. Los puntos sefialados anteriormente se refieren principalmente al aspecto producción u oferta. Nada se ha dicho, sin embargo, sobre

2. Decimos déficit concretamente porque, descontando las transferencias, no es muy realista pensar que en el mediano plazo El Salvador pueda revertir la situación de la cuenta corriente de su balanza de pagos. 
hacia dónde deberla de dirigirse esa producción, o sobre el aspecto demanda. A este respecto, se puede afimar que el mercado externo deberla jugar un papel fundamental; es decir, se tratarla de una estrategia de crecimiento con una orientación hacia afuera. Esta orientación no debe entenderse como el poner todo nuestro aparato productivo en función de las necesidades extemas. Más adelante se senala con mayor precisión qué entendemos por tal orientación. Por ahora, basta con apuntar que las exportaciones habrlan de constituir un elemento importante en el proceso de crecimiento. Sin pretender en modo alguno agotarlas, pueden senalarse dos razones importantes en favor de ello:

- La necesidad de hacer viable en términos macroeconómicos la estrategia de crecimiento (evitar el estrangulamiento extemo);

- La estrechez del mercado nacional y el límite que ésto impone al crecimiento.

Arriba nos hemos referido brevemente a la primera razón. Nos detendremos un poco en la segunda de ellas, que está vinculada especialmente con la producción manufacturera. La estrechez del mercado se define sobre todo con relación a lo que constituye el tamano óptimo mínimo de una planta industrial en la inmensa mayoría de actividades manufactureras ${ }^{3}$. Si un crecimiento fuerte exige el logro de una mayor eficiencia y productividad, éste es un factor de gran importancia. La necesidad de exportar resulta, en este sentido, una especie de corolario o consecuencia necesaria del uso de tecnología moderna altamente productiva y de un mercado intemo estrecho.

La necesidad de la integración regional aparece en este contexto, sobre todo si tenemos en cuenta que que ésta constituye una forma de ensanchar el mercado para nuestros productos. Cabe sefialar que una orientación hacia afuera no excluye en modo alguno una mayor integración a bloques comerciales regionales. De hecho, el destinar una buena parte de la producción a un mercado regional -producto o no de un esfuerzo de integración- no es sino una modalidad de orientación hacia afuera. Existe evidencia, sin embargo, de que aún la dimensión del mercado centroamericano resulta demasiado pequena - ex-

3. Sobre este punto, cfr. Carlos Mackinlay (1986). "Estructuras productivas y tamaño de las economias: el caso de las pequeñas economias latinoamericanas". Economla de América Latina Ne 15, pp. 137-210. Un estudio de un carácter distinto sobre el mismo tema es Frederic Pryor (1972). "The Size of Production Establishments in Manufacturing". Economic Joumal (Junio), pp. 547-566. 
cepto para unas pocas ramas industriales - para aprovechar las economlas de escala inherentes a gran cantidad de actividades manufactureras.4 Naturalmente, esto no significa que la integración centroamericana sea estéril como mecanismo para ampliar el mercado para nuestra producción industrial; significa que es necesario pensar también en los mercados extrarregionales como destino para al menos una parte de esa producción, a fin de tener al menos la posibilidad de aprovechar las economias de escala en la producción industrial.

+ Se ha hablado mucho de la necesidad de una orientación hacia adentro de la economla para dinamizar el proceso de crecimiento. Es preciso senalar, sin embargo, que cuando el tamano de la economla en términos de ingreso es tan pequeno, el volcarse hacia adentro se enfrenta inmediatamente con el tamano del mercado, a menos que, una vez se adopta el uso de tecnología (industrial) modema, se quiera renunciar a los beneficios que ésta ofrece en términos de productividad. Esto obviamente puede hacerse. El problema está en que tal renuncia significa sacrificar innecesariamente y en diversos grados el tamaño mismo del producto e incluso el logro de mayores niveles de empleo, y, de ese modo, también un crecimiento más rápido.

En El Salvador, en el pasado se ha aceptado en alguna medida tales sacrificios, manteniendo niveles elevados de capacidad ociosa en el sector industrial, aún con el mercado más amplio que la integración centroamericana ha posibilitado. ${ }^{5}$ En aquellas actividades en que son importantes las economias de escala, éso ha producido resultados desfavorables en términos de elevación de los costos de producción. ${ }^{6}$ Ha sido el consumidor quien, en último término, ha cargado con tales resultados a través de precios más altos, pues la elevada protección arancelaria ha garantizado a muchas actividades una especie de cautiverio del mercado doméstico.

4. Véase W.R. Cline (1982). "Las economlas de escala y la integración económica en América Latina". Integración Latinoamericana 7, \# 68 (Mayo), pp. 3-33. En este estudio se compara la escala óptima minima con el tamaño del mercado para veinte actividades manufactureras. El autor encuentra que el mercado salavadoreño en 1978 era lo suficientemente amplio sólo para una actividad (tejidos de algodón y sintéticos), mientras el centroamericano lo era únicamente para tres (tejidos de algodón y sintéticos, zapatos y harina) (Cuadros II-3 y A-4, pp. 15 y 31) .

5. Cfr. SIECA (1973). El desarrollo integrado de Centroamérica en la presente década. Buenos Aires: BID/INTAL; Blanca Cárcamo et. al. (1989). Capacidad ociosa en el sector industrial salvadoreño y reactivación económica. San Salvador: UCA. (Tesis de grado, economla). 
+ Es sumamente importante senalar que la orientación hacia ahuera de un patrón de crecimiento no es necesariamente incompatible con el imperativo de satisfacción de necesidades básicas, como su orientación hacia adentro no es en modo alguno garantla de mayores posibilidades de satisfacción de esas necesidades, en particular de las alimentarias. ${ }^{7}$ En principio, aparte de sus diferencias en cuanto a capacidad para hacer crecer más rápidamente el producto, no hay argumentos sólidos que respalden la idea de que uno es superior al otro en términos de favorecer la satisfacción de necesidades básicas. Este serla el caso si uno generara, por su misma naturaleza, un crecimiento más inequitativo que el otro. La evidencia mundial existente al respecto no es contundente, aunque pareciera inclinarse en favor del patrón de orienta ción hacia afuera. ${ }^{\text {En }}$ general, dado un cierto nivel del ingreso, la posibilidad de una mayor satisfacción de esas necesidades depende fundamentalmente de la acción distributiva y redistributiva propiciada por el Estado y de la estructura de distribución del poder dentro de la sociedad, más que del tipo de orientación del patrón de crecimiento. La experiencia de la inmensa mayoria de paises capitalistas que durante este siglo ensayaron un crecimiento industrial basado fundamentalmente en el mercado interno (o regional) parece decir bastante a este último respecto.

Por otra parte, la realidad de la inmensa mayoria de paises pequenos desarrollados nos muestra que su crecimiento depende fuertemente de sus exportaciones, ${ }^{9}$ y no por éso son incapaces de satisfacer las necesidades internas de consumo. No obstante, estos paises cuenta con un mercado interno fuerte, lo cual, sin embargo, no es fruto del volcarse hacia adentro, sino de que el tamano de sus economias es to suficientemente grande (su ingreso es to suficientemente alto). Países como Holanda, Bélgica, Luxemburgo, Suiza, etc. demuestran que si una economia es capaz de competir en el marcado mundial, si sus exportaciones son to suficientemente diversificadas, su nivel de desempleo lo suficientemente bajo y su ingreso está distribuido de

6. Véase Cline, op. cit., cuadro II-5.

7. Hacemos referencia especificamente a las necesidades alimentarias porque dentro de los bienes y servicios básicos son los alimentos y otros bienes similares los que son objeto del comercio internacional, y en los cuales se presenta realmente la opción de producirlos internamente, importarlos, etc.

8. Véase Banco Mundial (1987). Informe sobre el desarrollo mundial 1987. Nueva York: Oxford University Press.

9. Cfr. Vicente Galbis (1990). Economlas pequeñas y abiertas: estrategias comparadas. Mimeo. 
manera bastante equitativa, y es lo suficientemente alto, la dependencia del mercado internacional tanto en exportaciones como en importaciones (o una gran apertura externa) no representa en si un serio problema, ni en términos de vulnerabilidad ni en términos de satisfacción de necesidades básicas, o de seguridad alimentaria.

+ Si el problema fuera la seguridad alimentaria, en el sentido de asegurar a la población el acceso a una oferta interna "suficiente" de alimentos, ésto no ha de ser fruto necesariamente del volcarse hacia adentro, sino de que toda la población (o su inmensa mayorla) cuente con un ingreso que le permita satisfacer esas necesidades, teniendo en cuenta que también hay que satisfacer otras. Este tipo de seguridad no está asociado forzosamente a la orientación específica (hacia afuera o hacia adentro) de un patrón de crecimiento, sino al tamano del ingreso por habitante de la economía en cuestión, a la distribución de ese ingreso entre la población y a la capacidad doméstica de importación.

Un pals puede muy bien producir una oferta alimentaria suficiente para dar de comer a toda su población, pero si una (buena) parte de ésta no puede acceder a ella, esa autosuficiencia no sirve de mucho, o no sirve a los propósitos que debería servir. Lo que se sucederá, en ese caso, es que una parte de esa oferta se exportará, si se es lo suficienternente competitivo. Si no se es competitivo y la producción tampoco puede realizarse internamente, la producción alimentaria simplemente declinará y la autosuficiencia desaparecerá, tarde o temprano. ${ }^{10}$

De manera similar, un pals con un patrón de crecimiento orientado hacia afuera y deficitario en términos de producción alimentaria; pero cuya población (su inmesa mayoria al menos) cuenta con un nivel de ingreso to suficientemente alto como para satisfacer adecuadamente sus necesidades de alimentación, puede muy bien importar una parte de sus alimentos, y obviamente no estará en problemas. Una variedad de palses pequehos desarrollados ilustran muy bien ésto. Obviamente, siempre existe la posibilidad de una dependencia extrema de un pais determinado, y de que - por cualquier razón (una guerra, por ejernplo) - en un momento determinado no podamos importar de ese pais y esternos en serios problemas. Sin embargo, son sumamente escasos -si es que los hay- los bienes alimenticios que son producidos por un sólo pals o por un número muy reducido de paises para que esa

10. Esto serla inevitable, a menos que estemos pensando en una economla centralmente planificada y/o altamente subsidiada. 
posibilidad haya de ser tomada tan en serio, y decidamos lanzamos a la autarquia alimentaria."

+ En realidad, y en general, aquellos bienes que pueden o podrlan producirse internamente sólo a costas de grandes ineficiencias u otros costos (ecologicos, por ejemplo) -debido sobre todo a condiciones naturales o vinculadas con la naturaleza-, y que pueden ser comerciados internacionalmente, resulta más conveniente importarlos; a menos que se trate de bienes de gran valor estratégico y cuyo comercio internacional no sea tan generalizado más allá de cierto espacio geográfico (como la electricidad, por ejemplo). ${ }^{12}$

11. Lo anterior no significa que se abogue por una desprotección de la producción alimentaria de origen agropecuario. Significa solamente que la competitividad de esa producción en nuestro mercado no deberla fundarso en la mantención permamente de aranceles sumamente altos o prohibitivos. Por cierto, resulta un tanto paradójico que la estrategia de sustitución de importaciones basada on el mercado interno (y regional), seguida en El Salvador luego de la postguerra, penalizará fuertemente a ese tipo de producción en términos de protección arancolaria. Un régimen comercial más o menos neutral, como el que estarla tras una orientación hacia fuera, no necesitarla incurrir en tal penalización. $Y$ puesto que tal régimen no es sinónimo en modo alguno de desmantelamiento de la protección, la producción agropecuaria en general gozarla de una mayor protección que la que en el pasado ha tenido.

12. Para el caso de El Salvador, al menos, otra excepción importante la constituirla la producción de granos básicos, realizada on su gran mayorla por pequeños productores, quienes no sólo tienen en ella una fuente de vida importante, sino también prácticamente una cultura on tomo a la misma. En ese sentido, si fuera necosario, deberla de otorgarso un tratamiento especial --preferiblemente temporal- en tórminos de protección, pero más como una modalidad de polltica de ingresos que de política de autosuficiencia.

Por otra parte, debe tenerse en cuenta que la recionalldad de estos productores es muy distinta a del productor capitalista, y sus ectividades no pueden ser juzgadas, en términos de eficiencia, a partir de los mismo parámetros con que se juzgarla la eficiencia de la producción capitalista. De ahl que esa producción esté al margen del criterio arriba sohalado. Además, este es un sector con una muy baja productividad, no fundamentalmente por los caprichos de la naturaleza, sino porque la tecnologla, la infraestructura, asistencia téenica, crédito, etc. al que los productores tiene acceso es o muy pobre o inexistente. Como on otras actividades on el sector agropecuario, un esfuerzo serio de política sectorial tendiente a elevar la productividad de la producción de granos básicos serla imprescindible en el marco de una reconversión técnica del sector agropecuario. El resultado muy bien podrla ser que tal actividad mejore sustancialmente 
Nuestra posición es que, en general, debería producirse internamente lo que un país puede producir de manera eficiente, asi como lo que podría producir eficientemente y es necesario para la configuración de una estructura productiva que pueda respaldar firmemente un crecimiento sostenible en el tiempo. Esto último busca hacer referencia a las ventajas comparativas dinámicas, o a la necesidad o conveniencia de construir ventajas comparativas en determinados áreas - actividades (por ejemplo, en algunas áreas de la manufactura e incluso en algunos bienes alimenticios), de manera que no sean las ventajas comparativas estáticas o las fundadas en factores naturales las que dicten la forma que va a asumir la estructura productiva doméstica. Evidentemente, el mercado a secas no puede construir ventajas comparativas, como tampoco puede percibir o identificar satisfactoriamente las ventajas comparativas dinámicas. El Estado, junto con ciertas instancias de la sociedad civil, estaria llamado a jugar un rol importante en esa identíficación, y en la formulación e implementación de una política industrial de respaldo sistemático y deliberado a aquellas actividades en que las ventajes comparativas deben constuirse.

+ Más que la orientación hacia afuera, el problema está en qué tipo de bienes se deberia de exportar, en la diversificación de la exportaciones y en la forma en que éstas deberian de ser fomentadas. A este respecto, existen fuertes argumentos en contra de que un pais como El Salvador se especialice en exportaciones de origen agricola - sean éstas no tradicionales o tradicionales-, como propone la estrategia actualmente en marcha. Existen, por otra parte, grandes razones en favor del fomento a la exportación de cierto tipo de manufacturas ${ }^{13} \rightarrow \boldsymbol{y}$ en general de una industrialización eficiente y potenciadora de un crecimiento sostenible en el tiempo-, punto olvidado casi por completo en la actual estrategia. Asimismo, existen fuertes razones contra la idea

su competitividad, y que de hecho el país logre alcanzar una ventaja comparativa on ella, una ventaja comparativa construida sobre bases sólidas.

13. Otra vez, se presenta el problema de nuestra competitividad y de nuestra capacidad para producir eficiente y sistemáticamente ese tipo de bienes -y la consiguiente necesidad de la transformación productiva--, pues no contamos con la base industrial que ello requerirla. Esta es una de las áreas en que debe pensarse en la construcción de ventajas comparativas, por lo menos en aquellas actividades con el mayor potencial en el comercio internacional. La generalidad de las actividades de maquila que on la actual estrategia se fomentan, cumplen muy raras veces con ese criterio. 
de mejorar la competitividad de las exportaciones sobre la base de salarios reales bajos.

+ Es necesario senalar que la orientación hacia afuera del crecimiento no significa la renuncia a un proceso de sustitución de importaciones ni mucho menos que ésto carezca de toda importancia. La misma necesidad de hacer viable el sector externo es un factor que exige éso, y las ganancias en competitividad resultantes de la transformación productiva un factor que ayudarla a posibilitarlo. Tampoco significa que se tiene inevitablemente que retroceder en términos del grado de suficiencia alimentaria; ni que ramas vinculadas a la satisfacción de necesidades básicas no sean fortalecidas y no puedan contribuir a un mayor dinamismo económico, o que tengan necesariamente que declinar simplemente porque no podemos ser autosuficientes; si en esas ramas somos to suficientemente competitivos (o eficientes) o podemos llegar a serlo sobre bases auténticas, es decir, sin tener que recurrir a una potección desmesurada o permanente, a la degradación de los salarios reales, al deterioro del ecosistema, etc., no hay razón alguna para que éso deba suceder, y puede muy bien suceder lo contrario.

Tampoco significa descartar el mercado interno como fuente de dinamismo de la economía. El mercado intemo como fuente de dinamismo irá cobrando cada vez mayor importancia a medida que el ingreso se expanda. Este es un punto importante que debe recalcarse: el mercado interno no se fortalece simplemente destinando la producción hacia su interior, bajo el amparo de una elevada protección arancelaria y/o de subsidios; su fortalecimiento ha de provenir principalmente del crecimiento del tamano de la economia. Esto tampoco niega que ciertas medidas que afecten la distribución del ingreso no sean capaces de contribuir también a ese fortalecimiento, aunque sin aumentos significativos en el tamano de la economía estas medidas probablemente no tendrian en esa área un impacto muy importante. ${ }^{14}$ Si se harian sentir más en la estructura de la demanda interna y de la producción, asi como en la incidencia de la pobreza (contribuirlan sin duda alguna a disminuir la pobreza), particularmente desde una perspectiva estática.

En términos de política económica, la orientación hacia afuera significa, desde una perspectiva global, que debe disenarse y contarse con una política macroeconómica que por lo menos no penalice a las

14. Los resultados de la investigación de Cline, citados arriba, son bastante sugerentes a este respecto. Véase la nota 4. 
exportaciones con relación a las ventas en el mercado doméstico (contar con un régimen comercial más o menos neutral) ${ }^{15}$, y con una política sectorial que favorezca a las exportaciones, en términos de esfuerzos institucionales que, por ejemplo, faciliten el acceso o penetración de mercados, promuevan la elevación de la productividad de las actividades que producen bienes objeto del comercio internacional, etc.

Finalmente, la idea de orientar la economía hacia afuera en el sentido que acá nos interesa, no significa sino asumir como un problema básico la restricción externa -y por tanto asumir el fortalecimiento de la capacidad exportadora de la economía como pilar estratégico para un crecimiento sostenible en el largo plazo-, y asumir como restricción interna a un crecimiento fuerte, el tamano del mercado doméstico. Para fortalecer la capacidad exportadora nos parece fundamental el adoptar el objetivo de producir eficientemente tanto para el mercado internacional como para el mercado interno, y para obviar esa restricción interna la necesidad de destinar una buena parte de nuestra producción al mercado externo. Ambos elementos requieren de la adopción de una política económica como la arriba descrita. Es precisamente en estas líneas que tomaria sentido la orientación hacia afuera.

3. En las páginas precedentes hemos hecho referencia varias veces a la necesidad de la acción del Estado en algunos aspectos importantes dentro de lo que podria ser una estrategia de crecimiento. Es preciso senalar, sin embargo, que existe cierto grado de consenso en torno a que el Estado parece haber perdido capacidad para orientar eficientemente el proceso de desarrollo. Esta pérdida de capacidad resulta en gran medida de una pérdida de legitimidad, de su incapacidad de transformar las demandas societales en opciones políticas, del deterioro de su capacidad técnico-administrativa como resultado de la hipentrofia de su función de "empleador" de última instancia.

Sin embargo, la manifestación más evidente de esta pérdida de capacidad es la crisis fiscal. Diferentes factores, entre ellos el servicio de la deuda, el desproporcionado peso del gasto militar y la baja capacidad de recaudación tributaria, han contribuido a hacer aún más escasos los recursos públicos para el desarrollo. En este sentido, la crisis fiscal/crisis del Estado actuará como una restricción a superar, ya

15. Esto no significa necesariamente que el régimen comercial este desprovisto de toda selectividad. Este puede ser en términos globales neutral y al mismo tiempo mantener cierta selectividad, es decir, discriminar abiertamente en favor de ciertas actividades específicas. En nuestra opinión, esta selectividad debería de ser un componente fundamental de la estrategia. Esto también se aplica a la política sectorial. 
que no sólo será necesario aumentar la eficiencia del Estado, sino también sus recursos. Algún grado de redefinición de lo que tradicionalmente ha sido el rol del Estado en la economla se plantearla como necesario en ese contexto. Esa redefinición, sin embargo, no debe entenderse como la renuncia por parte del Estado a su rol de agente activo en la promoción del desarrollo económico y social.

\section{Distribuclón más equitatlva del Ingreso y reduceión de la pobreza}

No existe ninguna fuerza interna en el mecanismo del mercado que, partiendo de una muy alta desigualdad en la distribución del ingreso y de los activos sociales, y de un elevado déficit en la satisfacción de las necesidades de la población, oriente en forma automática la asignación de los recursos hacia la solución del problema de la pobreza y la desigualdad.

En tal sentido, una apropiación de los frutos del crecimiento por parte de los grupos de menores ingresos exige la adopción del objetivo de desarrollo equitativo entre los actores del espacio socio-politico.

Este desarrollo equitativo supone no sólo la mejora progresiva del bienestar material de la población en general, sino también una reducción de la desigualdad de los niveles de vida de los diferentes grupos sociales; es decir, un aumento más acelerado en los niveles de bienestar de aquellos grupos de menores ingresos, a fin avanzar hacia la erradicación de la pobreza que se constituye como la manifestación más flagrante de la desigualdad.

Sin pretender avalar el burocratismo y la hipertrofla estatal, es claro que en este esquema el Estado surge como el ente orientador de una estrategia de desarrollo equitativo. Ello porque el Estado, además de contar con más recursos para tratar de conciliar acumulación y distribución del ingreso, en una sociedad de alto conflicto social como la nuestra, es el actor con mayor capacidad de ordenar la pugna de intereses entre diferentes sectores de la sociedad que mantienen allos niveles de desconfianza y resentimiento mutuo.

La adopción del objetivo del desarrollo equitativo exige un esfuerzo sistemático del Estado en los campos de:

1. Pollticas redistributivas, que corrigen la distribución primaria mediante transferencias de ingresos o bienes y servicios;

2. Políticas distributivas, que afectan la distribución primaria del ingreso resultante de la estructura de propiedad y del funcionamiento del sisterna económico; 


\section{Pollticas selectivas de desarrollo.}

Nos referiremos acá a los dos primeros tipos de poltica.

1. Pollticas redistributivas: la intervención redistributlva del Estado se materializa en las llamadas politicas sociales, que buscan afectar el nivel de bienestar de las familias pobres mediante la provisión gratuita o subsidiada de ciertos bienes y/o servicios (educación, salud, alimentos, etc.). Estas pollticas están presentes en cualquier estilo de desarollo (por el grado de legitimidad que confieren) y varlan según el peso acordado a la equidad. Cuando se redefine la polltica social con el fin de crear las condiciones para que la ciudadanla se haga efectiva (la pobreza es la negación más elemental de los derechos del ciudadano). los medios instrumentales (gasto público social, seguridad social, etc.) pueden ser los mismos para distintas estrategias, pero los niveles de recursos movilizados, el grado de participación de los grupos y/o comunidades objetivo, asl como la selectividad (y/o el sesgo) del gasto público serlan muy diferentes.

En este campo juega un papel fundamental la política fiscal, tanto del lado del gasto (redistribución y satisfacción de necesidades básicas) como del lado de la tributación (promoción de la equidad tributaria, reducción desigualdad, estructura, ingresos y obtención recursos financieros), asl como el marco de regulación económica que orienta las relaciones inter e intra sectores económicos y sociales con el objeto de evitar contradicciones y agudización de conflictos.

2. Polfticas distributivas: dado que el trabajo constituye la principal y casi única fuente de ingreso de los pobres (por empleo asalariado 0 por autoempleo), la mejora de la cantidad y calidad de sus empleo y/o la mejora en la cantidad y calidad de sus activos productivos constituyen áreas claves para la superación de la pobreza y la promoción de una mayor igualdad en la distribución primaria del ingreso.

En tal sentido, el objetivo del desarrollo equitativo requiere de una adecuada politica laboral que permita la captura de parte de los frutos del crecimiento y del progreso técnico por parte de los sectores asalariados, asl como de una polltica que promueva no sólo la desconcentración en la estructura de propiedad y/o acceso a los activos productivos, sino también un incremento más acelerado en la cantidad, calidad de los activos de los pobres.

2.1. Polftica laboral: esta polltica englobarla las diferentes áreas de intervención del Estado en el mercado de trabajo y comprenderla diferentes aspectos vinculados a la política salarial, pollticas de generación de empleo, legislación laboral, etc. Evidentemente en una situa- 
ción de crisis como la que ha afectado a nuestro pals, la recuperación del poder adquisitivo de los salarios es una tarea prioritaria, al igual que la recuperación de la participación de los trabajadores en el ingreso total. Sin embargo, dado que el salario es un precio y como tal transmite información para la asignación de recursos en el largo plazo, no debe de abusarse de un manejo prioritariamente distributivo para no afectar los niveles de empleo, favoreciendo procesos intensivos en capital.

En tal sentido, es claro que uno de los aspectos claves es la búsqueda de mecanismos de formación y ajuste de los salarios en el corto plazo, que traten de conciliar la recuperación de los salarios reales con la estabilidad macroeconómica, y en el largo plazo vincular el aumento de los salarios reales a la ganancias de productividad (justicia distributiva).

Es necesario enfatizar que la política laboral no se reduce a la polltica salarial, y que para lograr una mayor participación de los trabajadores en el ingreso nacional influye mucho la calidad, la cantidad y estabilidad de los puestos ocupacionales creados (polltica de empleo). Por otra parte, siendo el mercado de trabajo un mercado altamente imperfecto en materia de los recursos de poder de los autores que en él intervienen, el fortalecimiento de la capacidad organizacional de los trabajadores influirá positivamente en la participación de los beneficios del crecimiento, fundamentalmente en las zonas rurales.

2.2. Politlcas orlentadas a aumentar la cantldad y productlvidad de los actlvos de los grupos de menores Ingresos: es evidente que la desigualdad en el acceso a la propiedad de activos flsicos (tierra y capital) y los diferenciales de productividad explican en gran medida la desigualdad observada en los ingresos personales (niveles de vida) de los distintos grupos sociales.

Por tanto, la redistribución estática de la riqueza (de los activos existentes) y/o la redistribución dinámica de la misma (roerientación de la inversión pública y acceso al crédito para favorecer una redistribución de los activos futuros) constituyen aspectos claves en aras a reducir la desigualdad y a erradicar la pobreza.

Sin embargo, dado su alto grado conflictual (por to menos para el caso de redistribución de los activos existentes), es necesario asegurar modalidades que garanticen la reasignación directa de los beneficios a los grupos más pobres, asi como el mayor efecto redistributivo neto (como ejemplo de una redistribución ineficiente podrlamos citar la nacionalización de la comercialización del café, ya que los excedentes de la actividad nunca fueron transferidos a los gnupos más pobres). 
No obstante, es incuestionable que si al origen de una alta concentración del ingreso encontramos una alta concentración de la propiedad, en su corrección juega un papel fundamental la redistribución de la tierra, un mayor acceso al crédito por parte de los pequenos productores y la orientación de la inversión pública para mejorar la calidad de los activos productivos de los gnupos de menores ingresos.

Evidentemente, la implementación de pollticas redistributivas supone una reorientación en la asignación de recursos que son escasos y un mayor papel del Estado. En tal sentido, exige también una mayor eficiencia y eficacia de la intervención pública (dadas las externalidades de dicha intervención), mediante un fortalecimiento de su capacidad técnico-administrativa y de su capacidad de formulación y planificación de política económica y social.

\section{Areas/temas de Investlgación}

1. Teniendo como marco las problemáticas generales de un crecimiento fuerte y sostenido y de volver más equitativa la distribución del ingreso en aras a avanzar en la erradicación de la pobreza, a partir de lo planteado en las páginas precedentes, se pueden identificar una serie de temas o áreas de investigación. Lo que a continuación se presenta no pretende ser en general títulos de posibles trabajos; se trata, más bien, de temas o áreas que pudieran o deberían ser abordados por el Instituto. Algunos de se ellos se traslapan y pudieran ser redundantes.

Es preciso insistir que lo que a continuación sigue se desprende del marco arriba planteado; los temas, en general, aunque pueden ser importantes en si mimos, adquieren verdadero sentido sólo en ese marco. Este debería ser el criterio principal en la identíficación de posibles temas/áreas a ser abordados por el Instituto.

2. El nivel de globalidad de los temas/áreas sugeridos es bastante diverso. Atendiendo a su globalidad, éstos han sido clasificados en cinco niveles, siendo el nivel $V$ el más global y el nivel I el menos global:

El nivel $V$ constituye to que podría denominarse el nivel de los temas "terminales", en el sentido de que su resultado proporcionaria una "imagen-objetivo" del proyecto económico deseado y del "patrón de desarrollo" que puede materializarlo. Evidentemente, la imagen objetivo resulta de aproximaciones sucesivas entre el diagnóstico (o diagnósticos) de la realidad sobre la que se pretende actuar y la imagen preliminar de sociedad deseada, que surge del criterio de demarcación adoptado para juzgar la realidad y/o los diferentes proyectos sociales. 
Los niveles IV y III están constituidos por los temas destinados a evaluar las opciones estratégicas y/o variantes de una misma estrategia que pueden ser capaces de transformar progresivamente la realidad (situación inicial de referencia) hasta la situación deseada.

Por último, los niveles II y I comprenden aquellos temas que examinan aspectos especlficos del funcionamiento de la economla nacional (y/o regional), del tipo de intervención pública y la relación finesmedios en materia de política económica, con el objeto de sustentar la formulación de proposiciones coherentes, tanto en el campo de las opciones estratégicas como en el campo de la polltica econórnica y social.

La breve descripción que acompana a los temas muestra que muchos de éstos deberian ser abordados con una orientación bastante fuerte de política económica/política de desarrollo, planteando, desde diversos grados de generalidad, elementos propositivos.

3. Los temas/áreas seleccionados son los siguientes:

\section{Nivel V}

1. Hacia una estrategia de enradicación de la pobreza en EI Salvador.

Este es un tema sumamente amplio y deberla a ser tratado a un nivel bastante global; trataría sobre la problemática vinculada a la necesidad de un crecimiento fuerte y sostenido, y de una distribución más equitativa del ingreso para enfrentar la pobreza en el pals. Este tema pudiera muy bien engblobar a los temas 4 y 5 . El trabajo debería ir acompanado de un diagnóstico sobre la magnitud y causas de la pobreza en El Salvador.

Al tema también podría dársele un tratamiento diacrónico. En una primera parte podría elaborarse un diagnóstico de la economia salvadorena, que juntamente con los criterios de demarcación adoptados proporcionaría un marco de referencia (o imagen preliminar) que guiaría las investigaciones a desarrollar. En un segundo momento se irian elaborando las bases para la finalización del proyecto (propuesta global alternativa) a través de los resultados de los análisis más específicos de la realidad nacional.

\section{Nivel IV}

2. Estrategias alternativas de crecimiento económico y su relación con la satisfacción de necesidades básicas. 
Este trabajo trataria sobre la relación existente entre crecimiento hacia adentro-satisfacción de necesidades y crecimiento hacia afuera-satisfacción de necesidades. Se discutirían las ventajas y desventajas de cada modelo de cara a posiblitar un grado mayor de satisfacción de tales necesidades. El trabajo ampliaría, profundizaría e introduciría nuevos elementos a la discusión que al respecto se ha esbozado atrás.

3. Reinserción de El Salvador en la economía mundial

A luz del examen de los principales cambios operados en la economía mundial y de las tendencias previsibles, se analizarlan las capacidades de la economía salvadoreha para reinsertarse dinámicamente en la economía mundial. El tema es complementario al tema 10, pero hace énfasis en el examen de escenarios y capacidades.

\section{Hacia una estrategia de crecimiento económico en El Salvador}

Este tema debería o podria abordarse como parte del tema 1.

En un trabajo independiente, se trataria lo esbozado en la primera parte de este documento.

5. Hacia una mayor equidad económico-social en El Salvador

Este tema también deberia o podría abordarse como parte del tema 1. Un trabajo separado sobre ésto abordaría desde una perspectiva global to planteado en la segunda parte de este documento en torno a una distribución más equitativa del ingreso y reducción de la pobreza. riedades

6. Equidad y crecimiento económico: conflictos y complementa-

El subtítulo lo dice todo: el trabajo trataría, por una parte, sobre los conflictos que podrían existir y que de hecho existen entre medidas tendientes al logro de una mayor equidad y aquellas que responden a la necesidad de una mayor acumulación, de transformación productiva, etc. para el logro y sostenimiento de un mayor crecimiento. Por otra parte, se abordarian también las complementariedades entre la equidad y el crecimiento, sobre todo las que se relacionan con la productividad/ eficiencia.

Nivel III

7. El ajuste estructural como estrategia de crecimiento

La idea sería evaluar esta estrategia de crecimiento, destacando tanto sus puntos favorables como sus limitaciones. El problema de la coherencia interna de la estrategia y de la secuencia con que generalmente se implementa seria uno de los punto importante a enjuiciar 
de cara a una crítica intema. En una critica extema se senalarlan las limitaciones 0 insuficiencias de la estrategia frente a la necesidad de contar con un crecimiento fuerte y sostenido, asl como su apoyo en varios aspectos importantes en un análisis de equilibrio parcial. Los resultados obtenidos podrian servir como insumos a las reflexiones que se desarrollarian en los temas 2 y 4.

8. La necesidad de transformación de la estructura productiva. Este tema puede dividirse a su vez al menos en 2:

- reconversión industrial

- reconversión técnica en sector agropecuario

Los trabajos estarlan enmarcados adentro de la necesidad de lograr un crecimiento fuerte y sostenido en el tiempo y de mejorar la productividad global y la capacidad productiva de la economla. La necesidad de la reconversión se vincularía también al imperativo de volver más competitiva nuestra economla, y en el caso de la reconversión industrial sobre todo, también a la apertura comerclal.

Los temas 11 y 21 estarlan bastante en esta linea.

9. Hacia una estrategia de promoción de exportaciones para un crecimiento fuerte y sostenido

El trabajo tendría como marco inmediato lo que se trate en el tema 3. Uno de los puntos principales a ser abordados en el trabajo sería el del modelo exportador a adoptar; es decir, qué tipo de blenes es más conveniente que un pais como El Salvador se dedique a exportar si ha de lograrse un crecimiento fuerte y sostenido. El como promover las exportaciones (los instrumentos y mecanismos para promover las exportaciones) seria otro problema abordado. Tamblén se trataría la necesidad de diversificación de las exportaciones y posibles políticas para avanzar en ese camino.

10. Variantes estratégicas de crecimiento hacla afuera: límites y potencialidades.

Se trataría de examinar las ventajas y/o desventajas de orientar el crecimiento hacia determinados mercados y/o bloques comerciales, as! como los efectos que tendría sobre el resto del aparato productivo (encadenamiento hacia atrás o hacia adelante) especializaciones sectoriales o subsectoriales de las exportaciones.

11. Apertura comercial y reestructuración Industrial: los nuevos desafios para la política industrial.

A pesar de la relativa industrialización desarrollada en EI Salvador en los sesenta y setenta, uno de las grandes limitaciones fue el bajo 
desempeno exportador del sector industrial. Sometidos a la necesidad de exponer la industria a la competencia internacional, en este tema se tratarian globalmente aspectos vinculados a la estrategia de configuración de microsistemas competitivos, desarrollo de interacciones entre pequenas y medianas empresas industriales con las grandes empresas, configuración de economias de escala y economías de "entorno institucional", patrones de especialización, etc. También se abordarian aspectos vinculados a las diversas prácticas y/o medidas de politica para desarrollo/reconversión industrial.

12. Precondiciones sociales para la configuración de un aparato productivo competitivo

Un aparato productivo es competitivo internacionalmente no únicamente por su base tecnológica, sino también $-y$ talvez principalmente- por el entorno socio-económico que lo respalda. En este sentido, se analizarian los efectos que sobre la competitividad podrian tener el nivel y forma de satisfacción de ciertas necesidades básicas vinculadas a la productividad de la mano de obra (educación, salud, alimentación), el grado de concertación existente en el espacio laboral, el grado de participación de la población en las decisiones de carácter social, etc.

13. Formas de integración de la economía informal con la economia moderna: posibilidades y/o limites para un crecimiento paralelo

La importancia del sector informal en la generación de ocupaciones es innegable; también es evidente que ese sector constituye un conjunto de actividades productivas heterogéneas (algunas arcaicas, otras funcionales al sector moderno de la economia) que desarrollan diversos grados de complementariedad-competencia con el sector moderno y que por tanto crecen y decrecen de acuerdo a la dinámica de este último. Este trabajo trataria de establecer globalmente las complementariedades existentes y las posibles (y-o deseables) entre ambos sectores, con el objeto de explorar soluciones a los problemas vinculados a la homogeneización de la estructura productiva, absorción y difusión acelerada de la innovación tecnológica, generación de empleo y crecimiento económico.

\section{Evaluación de políticas de reconversión industrial}

El trabajo desarrollaría un análisis de los diferentes instrumentos de política industrial que podrian ser utilizados para realizar la reconversión competitiva de nuestro aparato industrial, el grado de selectividad necesaria, los modelos de política (dirigista-subsidiaria), asi como la coherencia necesaria de las otra políticas económicas (comercial, mo- 
netaria). Este trabajo buscarla evaluar las acciones necosarias para transformar la estructura productiva poniendo énfasis en el sector industrial.

\section{Política macroeconómica y crecimiento con equidad}

Tratarla sobre la necesidad de contar con un contexto macroeconómico estable para el crecimiento, y el tipo de polltica macroeconómica que se requerirla, entendiendo por polltica macro tundamentalmente la politica financiera (monetario-crediticia, fiscal, cambiarla, financiera internacional). El problema de la establllzaclón económica en su relación con el crecimiento y la equidad, y de manera particular la necesidad de distribuir equitativamente los costos de abunas medidas de política macroeconómicas serla abordado en este trabajo. Se indagarla sobre alternativas posibles para una tal distribución de costos, sin poner bajo amenaza el proceso mismo de establlizaclón y la sustentabilidad del crecimiento.

16. Necesidad de modemización y fortalecimiento del sistema financiero

El trabajo se enmarcarla adentro de la necesidad de una mayor y mejor intermediación financiera como elemento necesario para el fortalecimiento del ahorro y la acumulación que un crecimiento fuerte y sostenido demanda. Se abordarlan temas como la conveniencia de un mercado de valores, el problema de los entes regulatorlos del sistema financiero, el problema de la liberalización financiera, el problema de la naturaleza de la propiedad bancaria, la banca de fomento, tasas de interés, etc.

El tema es sumamente amplio y puede muy bien ser abordado mediante varios trabajos (por lo menos más de uno).

17. El rol del financiamiento externo en el proceso de crecimiento económico

La idea serla discutir la necesidad del financiamiento externo para un crecimiento fuerte en una economla con un nivel tan bajo de ahorro nacional y que necesita entrar en un proceso de transformación productiva y volverse participe del progreso tecnológico mundlal. De ahl se derivarian subtemas como la inversión extranjera directa: sus ventajas y desventajas, el rol que podrla/deberla jugar, en que actividades puede ser más importante, el tratamiento que debe dársele, etc. También se tratarla en este marco el problema del endeudamiento extemo con un tratamiento similar al tema de la inversión extranjera. So abordarla también la cuestión de la ayuda extema y los efectos sobre el crecimiento de diferentes escenarios de restricción financiera. 
18. Necesidad de una estrategia de formación de capital humano

Tratarla precisamente sobre la necesidad de una estrategia de formación de capital humano para respaldar la transformación productiva nacional y potenciar el crecimiento económico en el mediano-largo plazo, asl como para la promoción de la equidad y combate directo a la pobreza. La aportación del capital humano al proceso de crecimiento y desarrollo serfa tratada. Los problemas de cuál es el tipo de educación que debe fomentarse y de la readecuación/reforma del sistema educativo seria abordados en un trabajo de esta naturaleza. El trabajo puede concluir ofreciendo lineamientos para una estrategia de formación de capital humano que responda a esa problemática.

19. Redefinición del papel del Estado en la economia

El trabajo podrla partir de la percepción existente con cierto grado de generalidad sobre la pérdida de la capacidad del Estado para orientar el proceso de crecimiento y desarrollo económico. Tratarla sobre las nuevas modalidades, mecanismo, áreas y criterios de intervención del Estado en la economia.

El problema institucional en general podrla también ser abordado.

20. Política tributaria, crecimiento y equidad

El trabajo puede pensarse dividido en 3 partes: en una primera se abordarla la relación entre politica tributaria y crecimiento; se discutirla sobre la politica tributaria más adecuada en aras a fomentar el ahorro, la inversión nacional y probablemente las exportaciones. Se discutirla también sobre la eficiencia económica de distintas altemativas tributarias. En una segunda parte se trataría la relación entre polftica tributaria y equidad, en un marco de redistribución del ingreso; es decir, cómo la politica tributaria puede ser un instrumento de promoción de la equidad, y cuál deberla ser la política tributaria más adecuada a ese respecto. En la tercer parte podría abordarse los conflictos potenciales que se presentan en una politica tributaria que ha de fomentar tanto el crecimiento como la equidad y cuál podrla ser una política tributaria (o sistema tributario) más adecuado desde esta perspectiva. De este tema este tema podría también incluir otros como:

- Evaluación de la capacidad redistributiva del Estado y restricción presupuestaria.

- Política tributaria: viabilidad de un financiamiento estable y equitativo de una acción pública fuerte y eficaz.

21. Modernización agrícola y reforma agraria: ¿conflicto o comple- 
mentariedad en el marco de la transformación productiva del sector agropecuario?

En una primera parte se trataría de establecer qué formas de organización institucional del sisterna agropecuario facilitan o bloquean la incorporación de innovaciones productivas, organizacionales y comerciales en el sector. Enfasis en evaluar la simple modemización agricolas vrs. una reforma agraria para determinar posibilidades de desarrollar ambas en el marco de un proceso de transformación productiva y desarrollo nural.

22. El rol de la politica social en el combate a la pobreza

En un trabajo sobre este temas se abordarlan problemas como los programas de educación, salud, vivienda, empleo, etc. y su contribución en el enfrentamiento del problema de la pobreza.

El problema de la polltica tributaria serla nuevamente abordado en alguna medida. Se ofrecerla también lineamientos generales para una política social altemativa.

23. El rol de la política laboral en el combate a la pobreza

El trabajo tendría como marco general la necesidad de una distribución verdaderamente equitativa de los frutos del crecimiento de cara la enfrentamiento del problema de la pobreza, sin amenazar el proceso de crecimiento mismo. Podría abordarse problemas como el de la necesidad de una organización adecuada para los trabajadores en general como instrumento para hacerlos participes permanentes de los frutos del crecimiento y de una mayor productividad. La definición de criterios para políticas salariales sería también un aspecto a considerar. El problema de la generalización de la seguridad eocial a todos los sectores de la economia, y la contribución de ello como instrumento de combate a la pobreza también sería tratado.

\section{La privatización}

Trataría sobre la naturaleza de la propiedad y la eficiencia en el uso de los recursos; el problema de la eficiencia en el sector público serla también abordado. Se tratarla también de establecer criterios más o menos específicos para la intervención estatal, asl como identificar áreas o actividades en que la presencia de la empresa privada serla más conveniente desde una óptica de eficiencia y crecimiento, sin que se presenten costos sociales de importancia, así como de aquellas en que el Estado no puede retirarse, con relativa independencia de los grados de eficiencia logrados. 


\section{Sustitución de importaciones y crecimiento hacia afuera}

Dado que el crecimiento hacia afuera implica transformaciones del aparato productivo y reorientación de la oferta industrial, en este trabajo se examinarian modalidades de industrialización que combinen eficientemente sustitución de importaciones y promoción de exportaciones, con el objeto de ganar mercado interno y mercado extemo en una "sustitución" industrial más global.

26. El papel de la integración centroamericana en el proceso de crecimiento.

El trabajo podría partir de una evaluación de las modalidades y esfuerzos de integración regional en el pasado; abordaria también las nuevas percepciones existentes en el área sobre las posibilidades y conveniencia de la misma, y los nuevos esfuerzos que en ese marco se vienen desarrollando. Luego se tratarla las modalidades que en el actual contexto regional y en el que se pertila para los próximos anos se presentan como las más viables, asi como las que debería asumir un esfuerzo renovado de integración. Se podría evaluar también la factibilidad y conveniencia de integrarse en una mayor medida a nuevos bloques comerciales regionales (México, El Caribe, por ejemplo).

27. Tecnología, crecimiento y empleo: el problema de la tecnología apropiada

El trabajo discutiria el dilema de la necesidad de incrementar la productividad en general cuando ésto implica un desplazamiento de mano de obra, y que por tanto aunque se contribuye al crecimiento se favorece muy poco la lucha contra el problema del desempleo. De esta manera se plantearia la necesidad de una tecnologia apropiada y en general de disenar y adoptar una política tecnológica. El problema de la desintegración del aparato productivo como uno de los elementos que favorecen la presencia de ese dilema sería también abordado. El problema las formas alternativas de promover una mayor industrialización también podría discutido en este contexto.

28. Evaluación del impacto redistributivo de las reformas estructurales.

Se analizaria (evaluaria) el efecto redistributivo de las reformas estructurales desarrolladas en los ochenta (en particular la reforma agraria) con el objeto de determinar su eficacia redistributiva y evaluar variantes estratégicas adentro del marco de continuación (profundización) de dichas reformas. 


\section{Apertura comercial y crecimiento económico}

Se tratarla de vincular la apertura al comercio exterior con la eficiencia global de la economfa (y por tanto con el crecimiento) y las posibilidades para la población de acceder más favorablemente a una determinada canasta de consumo. La necesidad de exponer la producción nacional a la competencia mundial como mecanismo para forzarla a una mayor eficiencia y para hacer partlcipe al pals del progreso técnico mundial sería discutida acá. La necesidad de lograr cierto avance en el proceso de transformación productlva (y en la competitividad) como precondición para la apertura extema serla un punto a abordar. El problema de los monopolios en una economla con un mercado nacional sumamente estrecho podrla ser abordado también acá, y cómo la apertura comercial pudiera hacer una contribución al respecto en algunas áreas industriales.

\section{Política cambiaria, polltica comercial y crecimlento}

El trabajo tratarla sobre la política cambiaria y comercial más adecuada a un crecimiento fuerte y sostenido. El enfoque estarla vinculado a la necesidad de promoción de las exportaciones y crecimiento orientado hacia afuera como forma de apoyar un crecimiento de esa naturaleza. Problemas como el del régimen cambiario, la relación entre tipo de cambio y estabilidad en una economía bastante abierta al comercio internacional; la política arancelaria y de gravación a las exportaciones, el ingreso al GATT, el problema de las excenciones y subsidios (directos/indirectos), las cuotas, etc. serían abordados acá.

\section{El problema agrario en El Salvador}

Se partirla del planteamiento de que si bien el crecimiento económico y la acción del Estado mediante la polltica social y redistribución dinámica son elementos imprescindibles en los esfuerzos de erradicación de la pobreza en el país, el origen ésta en gran medida vinculado a la estructura agraria, vuelven necesario la solución del problema agrario. El trabajo trataría sobre el problema agrario en general y su incidencia sobre la pobreza. En el se estudiaría también la estructura agraria tanto antes como después de la reforma de 1980. El trabajo plantearía la necesidad de profundización de la reforma agraria como condición necesaria para lograr avances significativos en un tiempo razonable en la erradicación de la pobreza, sobre todo en las zonas rurales.

Nivel II

32. La selectividad en las políticas crediticia y de tasas de interés 
Este tema estaría vinculado al de la liberalización financiera. Acá se evaluarla la conveniencia de la liberalización plena de las tasas de interés y del crédito frente a una manejo más dirigido de esas variables en el marco de una estrategia de desarrollo. Se trataria de dar respuesta a la interrogante de si no deben existir actividades privilegiadas que merezcan un tratamiento especial en términos de tasas de interés y acceso al crédito; o si por el contrario el Estado no debe inmiscuirse en absoluto en ese problema, que deberia quedar entonces en manos del mercado, atendiendo fundamentalmente al criterio de la rentabilidad bancaria.

\section{El problema de la seguridad alimentaria}

El trabajo abordaría la problemática de la seguridad alimentaria en El Salvador tanto desde la perspectiva de la oferta como de la demanda alimenticia. Desde la primera perspectiva, indagaría las posibilidades para un pais como El Salvador de alcanzar una seguridad alimentaria basada fundamentalmente en la producción interna. Desde la segunda, investigaría sobre los obstáculo que impiden a que la población en general pueda acceder a una canasta alimentaria mínimia en términos de cantidad y calidad.

34. El problema de la "democratización" del crédito.

Trataria en problema de la "democratización" como instrumento de distribución dinámica y de fomento de una mayor equidad, en aras a combatir la pobreza en general. Plantearia los problemas que supone una mayor democratización del crédito, asi como su necesidad y beneficios sociales. El problema de la banca de fomento tendria que ser abordado desde tal perspectiva en este trabajo; la necesidad de organizar la demanda de crédito, propiedad de la banca y democratización crediticia (o acceso más generalizado al crédito), etc. serían también puntos a abordar.

35. Pobreza y mercados de trabajo: análisis de los principales determinantes laborales de la reproducción de la pobreza.

Tal como lo indica el título, se abordaria en este trabajo el peso que tiene la posición del jefe de familia en la estructura ocupacional y la situación de pobreza de su familia. Igualmente, se analizarian aspectos vinculados a la relación movilización fuerza de trabajo familiar y pobreza. Este trabajo proporcionaría "insumos" para lineamientos de una política laboral alternativa. 
36. Impacto redistributivo de la seguridad social.

Se trataria de examinar el efecto redistributivo (o el impacto sobre los niveles de vida de las familias) de los actuales mecanismos de seguridad social, asl como examinar la capacidad de extenderla a toda la población trabajadora y de implementar nuevos mecanismos de compensación social.

37. Capacidad redistributiva del salario minimo y estructura salarial.

La fijación de salarios mínimos por parte del Estado responde, en teoria, al interés de éste por garantizar una remuneración capaz de garantizar la reproducción del trabajador y de su familia. Esta conquista laboral es aplicada únicamente a una proporción muy baja de la población asalariada. Se trataria, por tanto, de evaluar los mecanismos de formación del salario mínimo, el impacto redistributivo de elevarto y los posibles mecanismos altemativos capaces de cumplir con el objetivo de proporcionar un ingreso adecuado a las familias de los asalariados.

38. Crédito al sector informal en el marco de una estrategia de desarrollo equitativo

Este trabajo sería complementario al de "democratización del crédito", pero el énfasis estaría puesto en el papel que juega el crédito en el aumento de la capacidad de generación de ingresos para los pequenos productores. Además, deberá abordar aspectos vinculados a la selectividad del crédito, modalidades organizacionales, y diferenciación del crédito de otro tipo de ayudas de carácter más subsidiario.

39. Evaluación de los costos sociales de la reconversión industrial

Dado que la reconversión industrial implica el ajuste y la racionalización del sector, reducción de costos, introducción y modificación de técnicas de producción, etc. el proceso deberá indudablemente generar costos sociales asociados a la reducción de planillas en empresas "sobrevivientes", destrucción de empleos y quiebras empresariales, asi como costos públicos asociados a la reconversión laboral de los trabajadores cesantes. En este sentido, la evaluación de estos costos puede contribuir al desarrollo de acciones selectivas para la política de reconversión industrial.

\section{Nivel I}

40. El papel de la pequena empresa industrial en la reestructuración de sistemas industriales más competitivos

41. Evaluación del papel de la pequena propiedad agricola en el contexto de un desarrollo rural más equitativo 
42. Producción informal y demanda popular

43. Distribución del ingreso y grupos sociales

4. La lista de temas es bastante larga, y obviamente no puede ser agotada en el primer ano de trabajo del Instituto. De ahi que sea necesario establecer prioridades. En ésto debería considerarse tanto la importancia en si de los temas como su relevancia en el país en estos momentos, sobre todo si tenemos en cuenta que el Instituto, a la vez que pretende abordar problemas económicos fundamentales en EI Salvador, busca tener presencia en las discusiones que sobre la problemática económica se dan permanentemente en el pals.

Creemos que, en general, los temas que se adecúan más a esos propósitos son los que han sido clasificados en el nivel III. El tratamiento de estos temas, por otra parte, puede facilitar el abordar posteriormente temas más globales (algunos de los niveles IV y V), asi como algunos temas más específicos (niveles I y II).

Sin embargo, la lista de temas que corresponde al nivel III es todavia larga, por lo que habrá que hacer una mayor selección a fin de definir con mayor precisión un plan de trabajo para el Instituto. 